\title{
Development and applications of chip calorimeters as novel biosensors
}

This article was published in the following Dove Press journal:

Nanobiosensors in Disease Diagnosis

19 April 2012

Number of times this article has been viewed

\author{
Wonhee Lee ${ }^{1,2}$ \\ jinyoung Lee ${ }^{1,2}$ \\ Joonyoung Koh ${ }^{1,2}$ \\ 'Graduate School of Nanoscience \\ and Technology, Korea Advanced \\ Institute of Science and Technology \\ (KAIST), Daejeon, Republic of Korea; \\ ${ }^{2}$ KAIST Institute for the Nanocentury, \\ Daejeon, Republic of Korea
}

Correspondence: Wonhee Lee Graduate School of Nanoscience and Technology, KAIST, 29I Daehak-ro, Yuseong-Gu, Daejeon, 305-70I,

Republic of Korea

$\mathrm{Tel}+8242350$ III 7

Fax +8242350 II I0

Email whleeI53@kaist.ac.kr

\begin{abstract}
Calorimetry is the science of measuring heat. For more than 200 years now, calorimetry has advanced and various types of calorimeters have been developed for diverse applications. The development of microfabrication and microfluidics led to the advent of chip calorimeters, miniaturized calorimeters built and integrated as chips. Chip calorimeters, as labelfree biosensors, have many advantages due to their small sample volume and high-throughput capability. In this review, techniques for realizing chip calorimeters are discussed in terms of their major functional components: insulation, fluid handling, and thermometry. Recent trends in the development of chip calorimeters are also discussed, along with several application areas. New fabrication techniques can provide higher sensitivity and easier, more reliable sample handling for chip calorimeters, which would enable new application areas, such as the study of single cell metabolism.
\end{abstract}

Keywords: chip calorimeter, microfluidic biosensor, thermal sensor

\section{Introduction}

In 1783, Lavoisier invented the first calorimeter, called the ice calorimeter, which measured the heat generated from samples by measuring the amount of water that melted from the ice surrounding the samples. Since then, for more than 200 years now, many types of calorimeters have been invented and refined. Calorimeters are now essential tools for a wide range of scientific fields, from basic sciences such as biochemistry and high-energy physics to the pharmaceutical industry. ${ }^{1-4}$ For example, the isothermal titration calorimeter (ITC) and the differential scanning calorimeter (DSC) are extensively used in life science research for the thermodynamic characterization of molecular binding, and for studying the internal structure of biomolecules. The basis of calorimetric analysis, the measurement of heat, makes the calorimeter irreplaceable in many areas. To illustrate, the ITC is often called the "gold standard for measuring biomolecular interactions" because it can measure all binding parameters - stoichiometry, binding constant $(\mathrm{K})$, enthalpy $(\Delta \mathrm{H})$, free energy $(\Delta \mathrm{G})$, and entropy $(\Delta S)$ - in a single titration experiment.

From the viewpoint of sensor application, calorimetric sensing is unique for several reasons. A calorimetric sensor transduces heat (energy) or temperature change into an electric signal. This unique sensing mode has advantages and disadvantages. First, as heat is ubiquitous in nature and as almost all biological and chemical phenomena involve enthalpy changes, a calorimetric sensor can be used for virtually any type of interaction. However, this sensing mode has low sensitivity compared to other techniques, such as optical methods. Second, calorimetric sensing is label-free and 
does not require complicated sample preparations, such as antibody immobilization; it can detect the heat from insolution reactions without any modification. This advantage, however, leads to a disadvantage. As specific heat is indistinguishable, any nonspecific binding or unwanted reaction will result in a signal. Third, it is noninvasive (passive) sensing. For example, sensing metabolic heat from cells does not influence the cells, which allows further downstream analysis. Lastly, the sample properties (eg, opacity, porosity, phase) do not matter, which allows application to samples that are not compatible with other techniques, especially optical methods.

The advent of microfabrication and nanofabrication technologies allowed the miniaturization of the conventional bench-top scale instrument for the building of micro/ nanoelectromechanical systems ${ }^{5}$ or microfluidic embedded sensor systems, and ultimately, for achieving integrated systems, such as the micro total analysis system. ${ }^{6}$ Miniaturized sensors can provide many new properties and benefits, such as parallel operation, small characteristic dimensions, portability, and low power consumption. ${ }^{7}$ The need for high-throughput calorimetry in industry and in basic science has been a major driving force for the development of miniaturized calorimeters. ${ }^{8}$ The term chip calorimeter or integrated-circuit calorimeter has become widely used for a miniaturized calorimeter. In the pharmaceutical industry, calorimeters are used in several steps in the early stages of the small-molecule drug discovery process. For instance, calorimeters can be used in the development of a highthroughput assay for testing binding affinity and for the detailed characterization of the interactions of screened drug candidates. However, calorimeters cannot be directly used for high-throughput assays because of their long measurement time (typically 30 minutes); it would take several years to test tens of thousands of chemical compounds, even with an automated calorimeter running continuously. The thermal time constant of chip calorimeters has been reduced (typically in the order of 1 second and as small as 1 millisecond $^{9}$ ) and the measurement time can be significantly shorter. Moreover, chip calorimeter arrays can provide capability for hundreds of parallel calorimetric measurements, which brings the measurement throughput to a level higher by several orders of magnitude.

A chip calorimeter not only provides a solution for high-throughput measurement, but also provides distinct advantages over a large-scale calorimeter. First, as the device is small, it has a small heat capacity, which allows the reduction of the sample volume. Biological samples can be very costly and often cannot be produced in large amounts, making them impossible to analyze using large-scale calorimeters. Second, the device cost can be lowered by mass production. Disposable calorimetric sensor chips can be fabricated, which can reduce cross-contamination and can allow the safe disposal of hazardous samples. Regarding the cost of developing chip calorimeters, we can favorably compare it with other types of biosensors, such as electrochemical and optical sensors. Calorimetric sensors typically use thermocouples or thermistors as transduction components, which are basically simple electric circuits. Thermal and electrochemical sensors are easy to fabricate and use relatively inexpensive materials. Optical sensors, on the other hand, require more expensive and bulky components, such as lenses or lasers. Lastly, small devices with low power consumption can be used for field operations.

However, miniaturization also has disadvantages. A larger surface-to-volume ratio leads to substantial heat loss, and poor insulation decreases the device's sensitivity. Moreover, a smaller sample volume leads to a smaller amount of heat. When the amount of the sample is not a limiting factor, such as when the sample is abundant but its concentration is low, the use of large-scale calorimeters would be more appropriate.

In this review, the development and applications of chip calorimeters as novel biosensors are discussed. Some chip calorimeters have been developed to characterize the material properties of a small amount of solid samples. These are beyond the scope of this paper. Rather, chip calorimeters with fluid-handling capability are reviewed in terms of their major functional components: insulation, fluidic handling, and thermometry. Recent trends in the development of chip calorimeters are also discussed, according to their application areas.

\section{Background}

It is difficult to describe the structure and components of calorimeters in general terms because there are many methods of calorimetric measurement. As there are various types of calorimeters, there are many ways of classifying them. Table 1 shows the classification methods suggested by Hemminger and Höhne. ${ }^{10}$ For example, a typical ITC is a heat-compensating, isothermal, twin-measuring chamber calorimeter according to the classification. Although there are many different operation modes and measurement methods, most calorimeters have common components: a reaction chamber, a thermal sensor, and thermal insulation. A typical calorimeter using a liquid sample and its simplified model 
Table I Classification of calorimeters ${ }^{10}$

\begin{tabular}{ll}
\hline Primary criteria & Secondary criteria \\
\hline Principle of measurement & Heat compensation* \\
& Heat accumulating ${ }^{\dagger}$ \\
Hode of operation & Static \\
& Isothermal \\
& Isoperibol \\
& Adiabatic \\
& Dynamic \\
& Scanning of surrounding \\
& Isoperibol scanning \\
Construction principle & Adiabatic scanning \\
& Single measuring chamber \\
& Twin or differential measuring chamber \\
\hline
\end{tabular}

Notes: *Determination of the energy required for compensating the heat to be measured; †Measurement of the temperature change caused by the heat to be measured; $¥$ Measurement of the temperature difference between the sample and the surroundings caused by the heat to be measured.

are shown in Figures 1A and B. The calorimeter consists of a reaction chamber isolated from the environment (thermally and physically), a sample injection unit, and a thermal sensor (not shown in Figure 1). The energy released or absorbed within the reaction chamber is measured via sophisticated thermometry and electronics.

Meanwhile, there are not too many types of chip calorimeters, and it is relatively easy to describe them. A chip calorimeter is usually built on a membrane structure to provide thermal insulation (Figure 1C). Microfluidic channels can be built to deliver a sample and to define the reaction chamber (closed chamber). Otherwise, droplets of the sample are directly delivered on the sensing region (open chamber) with a micropipette or an inkjet. The reaction chamber (with an open or a closed configuration) is usually located at the center of the membrane to maximize the insulation. A thermometer is microfabricated on the sensing region, where reactions occur.

Figure 1B shows a model of a simple calorimeter. The model (without a dotted-line box) can be described by equation 1, where $C$ is the heat capacity of the sample, $G$ is the thermal conductance of the calorimeter, $P(t)$ is the power generated from the sample, and $T(t)$ and $T_{0}(t)$ are the temperatures of the sample and the environment, respectively. Equation 1 is also known as the heat balance equation of a simple body.

$$
C \frac{d T(t)}{d t}+G\left[T(t)-T_{0}(t)\right]=P(t)
$$

To apply this equation to a real calorimeter, $C$ and $G$ should be constant and not a function of time and temperature, and the temperature of the sample body should be uniform.
In reality, however, the sample temperature cannot always be uniform. Therefore, the insulation structure has to be designed so the temperature distribution in the sample chamber is relatively uniform compared to the other regions of the membrane. Otherwise, the measurement will be incorrect. Most chip calorimeters use the heat exchange mode, where the temperature difference between the sensing region and the heat sink (substrate) is measured. The power $(P)$ can be calculated if the heat capacity $(C)$ and thermal conductance $(G)$ are known. Typically, chip calorimeters are calibrated using an electrical heater integrated with the chip. The structural body of the calorimeter chamber gives additional heat capacity $\left(C_{\text {add }}\right)$ and thermal conductance $\left(G_{\text {add }}\right)$. A large portion of the efforts to develop chip calorimeters involves the reduction of $C_{\text {add }}$ and $G_{\text {add }}$ via thermal engineering to achieve better sensitivity.

\section{Development of chip calorimeters}

A chip calorimeter can be divided into three major functional components: thermal insulation, fluid handling, and a thermal sensor (including electronics). The strategies for building these components vary from one chip calorimeter to another, and each strategy has both advantages and disadvantages. These strategies will be discussed in this section.

\section{Thermal insulation}

The reaction chamber is the central component of a calorimeter. It is where the sample mixing and reaction occur and where the temperature changes. Thus, the thermal insulation of the reaction chamber directly influences the sensitivity of the calorimeter. The thermal insulation of the chamber from the environment is especially challenging for a chip calorimeter due to its small sample volume and the large surface-to-volume ratio of the samples. There are four modes of heat transfer in the chip calorimeter system: conduction, convection, radiation, and evaporation of the sample. For a typical chip calorimeter configuration, conduction is the dominant heat transfer path due to the small length scale. ${ }^{11}$ The chip calorimeters that have been developed to date can be classified into two categories according to the configuration of their reaction chambers: the open-chamber (membrane) structure and the closedchamber (microfluidic channel) structure (Figure 1C). Both types of calorimeters use a microfabricated membrane as their main structural component, and reaction chambers and thermometers are built on the membrane. Openchamber chip calorimeters have well structures as reaction chambers or simply use the bare membranes onto which 

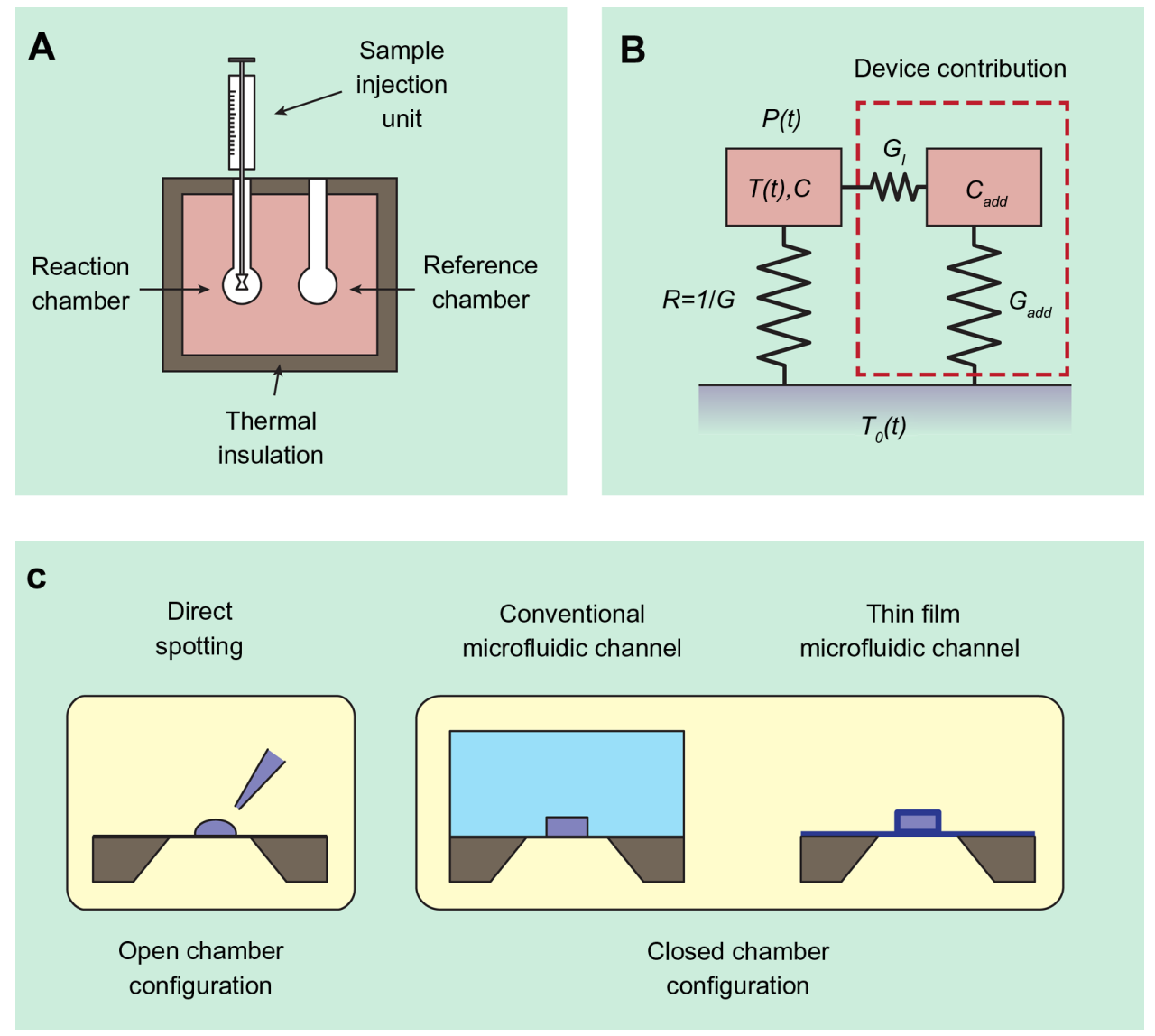

Figure I (A) Schematic of the typical bench-top calorimeter with twin measuring chambers. Major components include a sample-handling unit, calorimetric chambers, thermal insulation, and a thermometer (not shown). (B) A simple model of a calorimeter with an additional heat-loss path due to device contribution (dotted-line box). The ideal model (without device contribution) consists of three parts: the sample ( $C$ : heat capacity, $T(t)$ : temperature), the environment $\left(T_{0}(t)\right)$, and the thermal link between them (R: thermal resistance, $G$ : thermal conductance). The additional heat capacity $\left(C_{\text {add }}\right)$ and thermal conductance $\left(G_{\text {add }}\right)$ can result in poor insulation and lower sensitivity. Typically, the thermal conductance between sample and device $\left(G_{)}\right)$is much larger compared to $G_{\text {add }}$ and $G$. (C) Constructions of chip calorimeters according to the chamber type. Chip calorimeters have essentially the same components as large-scale calorimeters, but different approaches are used to build the components, especially the fluidhandling methods.

the samples are spotted as droplets by a micropipette or inkjet (Figures 1C, 2A, and B). ${ }^{9,12-25}$ Closed-chamber chip calorimeters, on the other hand, have microfluidic structures built on the membrane to guide the samples and to define the reaction chambers (Figures 1C, 2C, and D). ${ }^{26-43}$

In the case of the open-chamber chip calorimeters, the evaporation of the sample can significantly influence the measurements due to the lack of physical confinement. In fact, several early demonstrations of the operation of chip calorimeters showed large signals due to the evaporation of water droplets. ${ }^{9}{ }^{17}$ Evaporation should thus be prevented either by controlling the humidity or by sealing the calorimeter in a relatively small volume to saturate vaporization. ${ }^{25}$ Once the evaporation issue is properly managed, thermal conduction needs to be carefully engineered to achieve high sensitivity for the open-chamber chip calorimeters. The thermal conduction path includes the membrane, the electric leads, and air.
Thermal insulation is a bigger issue in the closed-chamber chip calorimeters because the additional materials for the fluidic channels can significantly deteriorate the thermal insulation (large $C_{\text {add }}$ and $G_{\text {add }}$ ). The fundamental problem with adding a microfluidic structure is that the conventional microfluidic channels are different from the miniaturization of large-scale tubing. The microfluidic structures are typically molded with polydimethylsiloxane (PDMS) or etched on an $\mathrm{Si}$ or $\mathrm{SiO}_{2}$ substrate. These structures can be compared to tunnels surrounded by huge mass, while most "macrofluidic channels" are pipes with thin walls. In addition to the large thermal conductance, the large heat capacity of the bulky device takes away the heat from the sample, which reduces the sensitivity and leads to a large sample volume requirement. A thin-film microfluidic structure using parylene can address this issue (Figures 1C and 2D). ${ }^{44,45}$ The parylene microfluidic structure is a realistic miniaturization of a large-scale calorimeter chamber that enables thermal insulation as good as that in the 


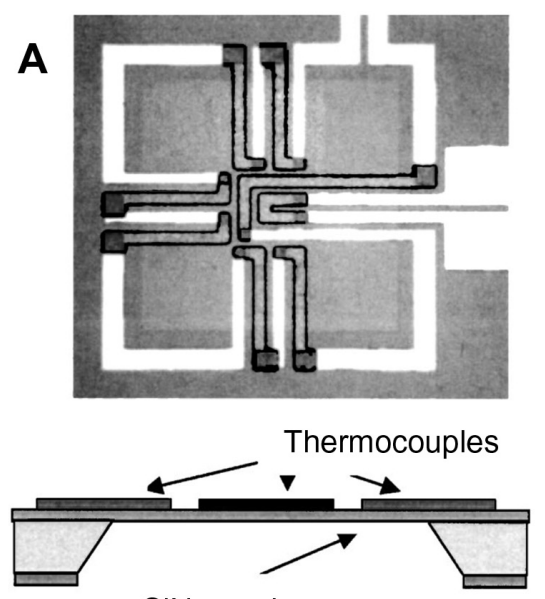

SiN membrane
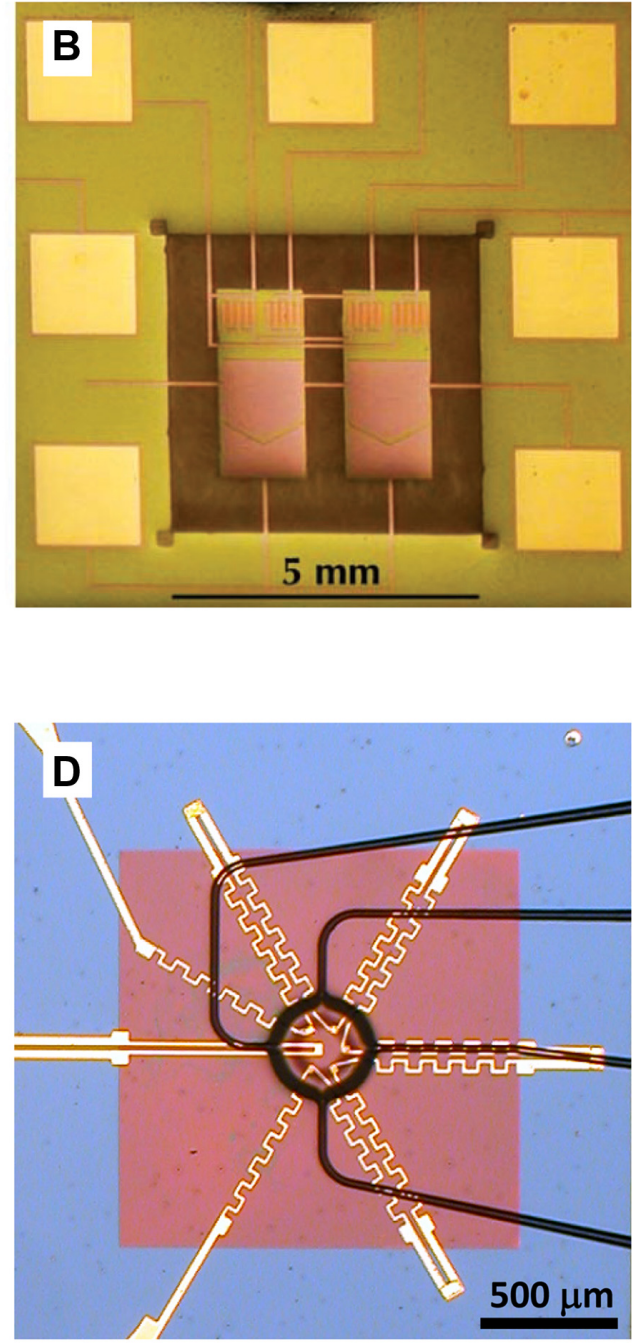

Figure 2 Examples of chip calorimeters. (A) The basic components of chip calorimeters are a thin membrane and a thermometer, and in this case, a Ti-Bi thermopile on an SiN membrane. Copyright (C) 2004, American Institute of Physics. Reproduced with permission from Chancellor EB, Wikswo JP, Baudenbacher F, Radparvar M, Osterman D. Heat conduction calorimeter for massively parallel high throughput measurements with picoliter sample volumes. Appl Phys Lett. 2004;85(I2):2408-24I0.9 Inkjet fluid delivery was proposed. (B) Droplets of the samples are spotted on the sensing pads and are electrostatically merged to avoid noise from the kinetic energy of the sample delivery. Copyright (C) 2004, National Academy of Sciences USA. Reproduced with permission from Torres FE, Kuhnt P, De Bruyker D, et al. Enthalpy arrays. Proc Natl Acad Sci U S A. 2004; I0I(26):9517-9522. ${ }^{22}$ Thermistors measure the differential temperature changes. (C) A PMMA fluidic channel is assembled on top of the thermopiles built on the membrane. Copyright (C) 2006, Elsevier. Reproduced with permission Lerchner J, Wolf A, Wolf G, et al. A new micro-fluid chip calorimeter for biochemical applications. Thermochim Acta. 2006;445(2): 144-150. ${ }^{34}$ Four consecutive thermopiles are used to sense the temperature change along the flows. The channel length, from inlet to outlet, is $20 \mathrm{~mm}$. (D) Parylene thin-film microfluidic channels and vacuum insulation enabled unprecedented high-sensitivity with $3.5 \mathrm{~nL}$ sample volume. ${ }^{31}$

Abbreviation: PMMA, poly(methylmethacrylate).

open-chamber chip calorimeter, and that prevents evaporation through the closed microfluidic-channel walls.

The engineering thermal properties of the device depend on the choice of material and the structural design, such as the shape and size. Table 2 shows the thermal conductivities of the materials that can be found in chip calorimeters. In most cases, the membrane is built with silicon or silicon dielectric materials $\left(\mathrm{SiN}, \mathrm{SiO}_{2}\right)$. A handful of calorimeters, however, utilize polymer membranes (SU-8, ${ }^{36}$ polyimide, ${ }^{28}$ parylene ${ }^{31}$ ) to enhance the thermal insulation. Si-based materials have relatively high conductivities, but they can have small thicknesses (a few hundred $\mathrm{nm}$ ) and provide fairly good insulation.
Polymer membranes typically have $\sim 100$ times lower thermal conductivities than Si-based materials, and are $\sim 10$ times thicker. Therefore, from the viewpoint of thermal insulation, polymer membranes are better than Si-based materials. They are, however, not always compatible with various microfabrication processes, which will make it difficult to mass produce the device. Electric leads for thermometry can also significantly contribute to the thermal conductance. There are trade-offs between the reduction of the thermal conductance and the other factors. Reducing the cross-section of the electric leads results in higher electrical resistance, and a thinning membrane can result in a mechanically fragile structure. 
Table 2 Thermal properties of the common materials used for chip calorimeters

\begin{tabular}{lll}
\hline Materials & $\begin{array}{l}\text { Thermal conductivity } \\
(\mathbf{W} / \mathbf{m} \cdot \mathbf{K})\end{array}$ & $\begin{array}{l}\text { Specific heat } \\
\mathbf{( J / g} \cdot \mathbf{K})\end{array}$ \\
\hline $\mathrm{SiN}(\mathrm{LPCVD})^{79}$ & $15 \sim 30$ & 0.7 \\
$\mathrm{Si}$ & $130^{80}$ & $\left.0.7\right|^{81}$ \\
Water $^{82}$ & 0.58 & 4.20 \\
PDMS $^{83}$ & 0.15 & 1.46 \\
Polyimide $^{84}$ & 0.12 & 1.09 \\
Parylene C $^{85}$ & 0.082 & 0.71 \\
Airr $^{86}$ & 0.026 & 1.01 \\
Stainless steel, $_{\text {austenitic grade }}^{31}$ & $11-16$ & $0.45-0.55$ \\
Gold $^{87}$ & & \\
Nickel $^{88}$ & 315 & 0.13 \\
\hline
\end{tabular}

As opposed to large-scale calorimeters, air is an important heat conduction path for chip calorimeters. Although air is a very good insulator, the thermal conductance it provides is a large portion of the total thermal conductance (eg, larger than $90 \%{ }^{31}$ ) due to the large heat loss from the surfaces of the samples. Further enhancement of thermal insulation can be achieved with a vacuum, which can provide the best possible insulation. Chip calorimeters using solid samples ${ }^{21}$ may be loaded in a vacuum chamber. However, liquid samples cannot be directly placed in a vacuum, which is most likely the case for biological applications. The combination of a vacuum and a microfluidic channel can address this issue. A microfluidic channel embedded in a vacuum was first invented for the resonator mass sensor. ${ }^{46}$ Here, cantilever-shaped microfluidic channels are used to remove large viscous damping. The use of a thin-film parylene microfluidic channel and vacuum encapsulation was a breakthrough in terms of the insulation issue affecting chip calorimeters (Figure 2D). ${ }^{31}$ Parylene microfluidic channels are strong enough to hold vacuum with only a few- $\mu$ m-thick layer, which also provides very low thermal conductivity. Despite its importance, the thermal conduction of the membrane and the electric leads were not particularly considered in the design rules for chip calorimeters because air or the microfluidic channel dominates the thermal conductance. It has become important to consider the balance of the thermal conductance, sensitivity, and stability of the device because the dominating heat loss paths can be removed and insulation can be maximized with vacuum-insulated microfluidic systems.

\section{Fluid handling}

The classification of chip calorimeters according to chamber configuration is also useful when considering fluid-handling techniques. For open-chamber chip calorimeters, samples with volumes ranging from a few $\mu \mathrm{L}$ to hundreds of $\mathrm{pL}$ are spotted as droplets on the sensing region. For closed-chamber chip calorimeters, the samples are flown through channels and are delivered to the sensing region.

For fluid handling in open-chamber chip calorimeters, samples are delivered via an inkjet or micropipettes. The inkjet sample delivery method can be useful when highthroughput fluid handling is required because it is very fast and has high spatial accuracy (Figure 2A). ${ }^{9}$ The large kinetic energy of the sample deposition, however, causes heat generation, which makes it hard for the inkjet to be used directly for chip calorimeters. Therefore, most open-chamber chip calorimeters use micropipettes for sample delivery. Micropipettes can also provide precise volume control, but are relatively slow and are hard to multiplex.

In general, fluid handling under the open-chamber geometry has the problem of unwanted signals from evaporation and kinetic energy. Slow and careful merging can reduce the kinetic energy, ${ }^{25}$ but it is not suitable for high-throughput calorimetry. In addition, a baseline shift was observed because configuration changes associated with sample merging will result in a change in the thermal conductance and the steady-state heating and evaporation ratio. ${ }^{19} \mathrm{~A}$ special fluid-handling method was developed to circumvent the kinetic energy issue (Figure 2B). ${ }^{22}$ Samples were first delivered to the sensing region using micropipettes. Then, when the samples had been thermally equilibrated, two droplets were merged electrostatically. By separating the sample delivery and merging processes, measurement error due to the kinetic energy was removed. This method also enhances the sample mixing. (Other fluid-mixing methods mostly rely on diffusion).

For closed-chamber chip calorimeters, samples are delivered through the tubing or microfluidic channels using a syringe pump or a peristaltic pump. The flow modes are either continuous injection $(\sim 10-00 \mu \mathrm{L} / \mathrm{min})$ or impulse injection $(\sim 10 \mu \mathrm{L})$. Impulse injections for closed-chamber chip calorimeters have large sample volumes due to the dead volume of their fluidic system. One exceptional example is a sample injection of $0.7 \mathrm{~nL}$ with on-chip pumps. ${ }^{31}$ Twolayer, on-chip, PDMS microfluidic pumps and valves can provide fairly accurate sample handling at the $\mathrm{pL}$ scale. Early realization of the closed-chamber chip calorimeter was achieved by the simple assembly of open-chamber chip calorimeters with gaskets and tubings to form reaction chambers. ${ }^{27,29,38,40,41}$ These large-scale tubings assembled with 
microscale chip calorimeters led to a large dead volume and a large heat loss. To reduce the sample consumption and to effectively measure the thermal event, the channel dimensions must be minimized and the channel must be localized within the sensing region. The development of microfluidics enabled the incorporation of microfluidic channels with chip calorimeters. Typically, microfluidic channels can first be molded with PDMS ${ }^{30,35,36}$ or etch-patterned onto glass ${ }^{39,43}$ and then bonded onto the membrane chip. Alternatively, the side walls of microfluidic channels can first be patterned with SU-8, and the tops of the channels can be sealed with PDMS ${ }^{42}$ or poly(methyl methacrylate) (Figure 2C). 26,33,34 Sample handling using a microfluidic channel has a negligible measurement error due to the evaporation and kinetic energy. It can be readily multiplexed for high-throughput applications. Biomolecules such as protein, however, can nonspecifically bind on the chamber surface and give a signal. The surfaces of microfluidic channels need to be treated in a way that will prevent nonspecific binding (eg, using poly(ethylene glycol)). Two-phase microfluidics using water droplets in oil can be an alternative solution.

\section{Thermometry}

The sensitivity of a calorimeter is determined by the minimum thermal energy or power it can detect. In most cases, calorimetric measurement is achieved by measuring temperature changes. Even for an isothermal calorimeter, very small temperature changes are measured, and the power that is needed to keep the temperature within the small variation is calculated. Therefore, a high-sensitivity thermometer is needed in developing a high-sensitivity calorimeter. Most room temperature thermometers use electrical methods such as thermocouples and thermistors, which is also true for chip calorimeters.

Thermopiles (thermocouples connected in series) are used as thermometers in most chip calorimeters. $9,12,14,21,23,25,27,28,31,3,3,3,6,2,43,47-54$ The thermocouple converts the temperature difference to electric voltage via thermoelectricity. As thermopiles can have very high sensitivity $(\Delta \mathrm{T}: \sim 10 \mu \mathrm{K})$ and do not use electric power to measure temperature, they are ideal for calorimetric applications. The thermoelectric signal-to-noise ratio can be improved by increasing the number of thermocouples $(n)$ in series (proportional to $\sqrt{n}$ ). There is a limitation, however, in increasing the sensitivity with series connection, not only because there is limited physical space but also because the connection of the thermocouple in series increases the electric resistance, which cannot be too large for electric measurement. Moreover, adding materials to the membrane leads to higher thermal conductance and heat capacity, which results in lower sensitivity. Thus, the electric signal-to-noise ratio increases and the thermal-insulation enhancement must be carefully balanced.

Thermistors are used for a handful of chip calorimeters. ${ }^{22,40}$ A thermistor measures temperature based on the fact that the electric resistance of a material depends on temperature. A thermistor can have very high sensitivity $(\Delta \mathrm{T}: \sim 10 \mu \mathrm{K})$ and needs no series connection, thus requiring a small space compared to the thermopile, which also helps reduce the thermal conductance. In addition, a thermistor can measure absolute temperature while a thermocouple can measure only relative temperature. However, thermistors need an electric current to measure the resistance, which leads to Joule heating and the increasing temperature of the reaction chamber. Hence, the heating effect must be considered when calibrating a chip calorimeter. A resistive thermal detector may be used instead of a thermistor. ${ }^{37}$ Resistive thermal detector thermometry has linearity over a wide range of temperatures, but has lower sensitivity $(\Delta \mathrm{T}: \sim 1 \mathrm{mK})$.

Thermometries other than the electric methods may be utilized for chip calorimeters. Many of these techniques do not require contact with the sample and thus offer better insulation. Infrared thermography and pyroelectric imaging ${ }^{55}$ are widely used in the biomedical field because they provide simple and cheap methods of measuring temperature. Infrared thermography has been used to monitor the thermogenesis in cell cultures, although the exact thermal power has not been measured. ${ }^{56}$ Infrared thermography and pyroelectric imaging, however, can measure only the surface temperature information and have low sensitivity $(\Delta \mathrm{T}$ : $\sim 0.1 \mathrm{~K}$ ). The interferometric technique has been used for temperature measurement within microfluidic channels. ${ }^{57}$ The temperature dependence of the refractive index change allows fairly sensitive thermometry $(\Delta \mathrm{T}: \sim 0.001 \mathrm{~K})$. This technique, however, is not suitable for calorimetric applications, where solution exchanges are involved. Similarly, optoacoustic methods have been developed to measure the temperatures of tissue samples. ${ }^{58,59}$ Interferometric measurement of the thermal expansion of a chamber has been used to build a chip calorimeter. ${ }^{60}$ Thermal-expansion thermometry reportedly has the highest sensitivity at room temperature $(\sim 2 \mu \mathrm{K}) \cdot{ }^{61}$ The size of the thermometer and sample are directly related to the sensitivity, and a relatively large sample volume $(\sim 10 \mu \mathrm{L})$ is required. Interferometric techniques also require mechanical stability for accurate measurements and any vibration or mechanical stress will result in measurement errors. Other thermometry techniques that can be used 
in the microfluidic setup include spectroscopic methods (eg, nuclear magnetic resonance, Raman spectroscopy, and temperature-sensitive molecular probes (eg, liquid crystals, fluorescent molecules). ${ }^{62}$

\section{Mode of operation}

While large-scale calorimeters have diverse modes of operation, chip calorimeters have only a few modes. All chip calorimeters have constant substrate temperature conditions (isoperibol mode), conditions where the heat of reaction changes the sample temperature (heat exchange or heat conduction mode), or conditions where the electric heating modulates the sample temperature (temperature-scanning mode). Heat exchange calorimeters can be classified into two types according to the sample handling process: batch and flow calorimeters. In this paper, chip calorimeters are categorized into three types: batch calorimeters, flow calorimeters, and scanning calorimeters.

Batch-type calorimetry is simple and is the operation mode

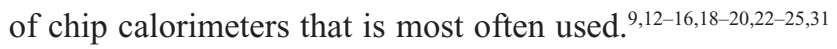
Specific amounts of the samples are delivered and mixed at the sensing region (reaction chamber), and the temperature changes are measured. Batch calorimeters are designed to measure the total thermal energy of a given volume, which can reveal the thermodynamic characteristics of the reactions. Titration chip calorimeters are similar to batch-type calorimeters. Several research groups are developing highthroughput, high-sensitivity chip calorimeters with minimal sample volumes. High-throughput measurements with enthalpy arrays have been demonstrated. ${ }^{19}$ Measurements of the heat of reaction from a few $\mathrm{nL}$ samples have been demonstrated within a fluidic channel (Figure 3). ${ }^{31}$ Titrations with a $10-n L$ sample have also been demonstrated. ${ }^{16}$

Flow calorimeters are typically operated with a constant substrate flow. ${ }^{26,27,29-35,37-43}$ The samples are delivered through the microfluidic channels or capillary tubings assembled over the sensing region. The sample flow can provide a continuous supply of samples, but it also results in extra heat capacity and heat loss. Therefore, the thermal insulation of the device itself can be less critical. As the flow operation consumes a large amount of samples, it is not suitable for the thermal characterization of a sample. This type of operation method is good for the continuous, real-time monitoring of a specific compound or the heat production of a living organism. Fluidic channels can be configured so that two samples can merge in the reaction chamber or one sample is flowed over the other sample immobilized in the reaction chamber. Biomolecules such as enzymes, antibodies, and DNA can be immobilized on the trapped microbeads $s^{35,48}$ or directly on the reaction chamber surface. ${ }^{27,38,40}$

A DSC measures the heat capacity change of a sample via temperature scanning, which allows the study of the internal structure or stability of biological systems in their natural state, without labeling. ${ }^{17,21,28,36}$ However, there are some technical difficulties in developing a chip calorimeter as a DSC. In general, a DSC requires a closed sample chamber

\section{A}

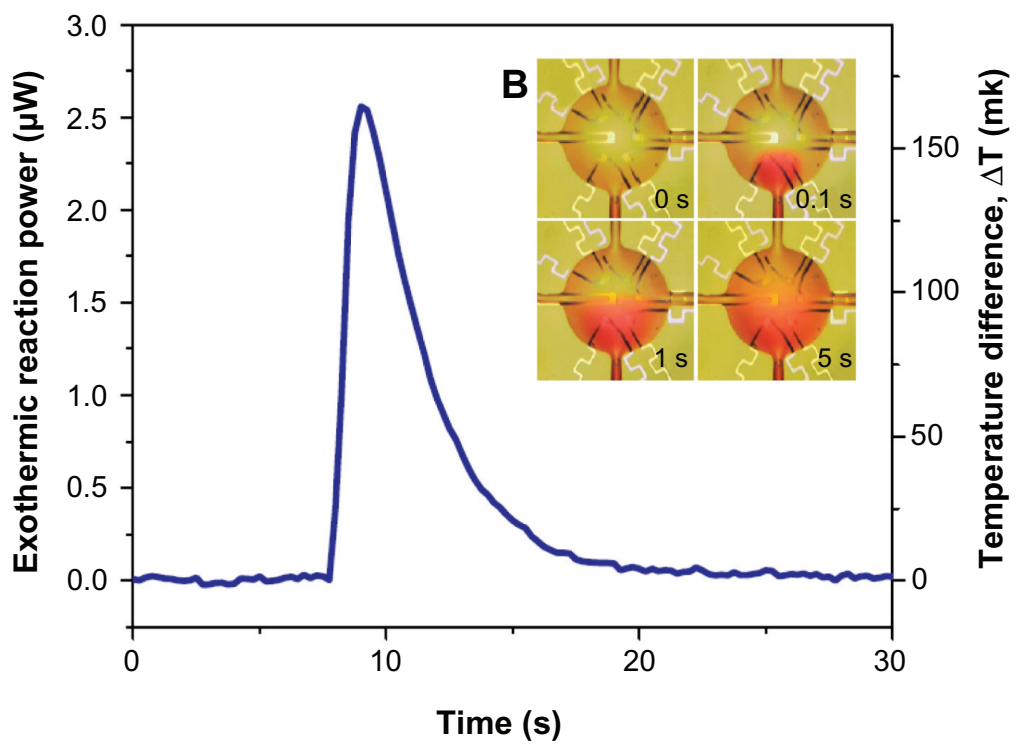

Figure 3 Chip calorimeters allow a small sample volume and a fast measurement time scale. ${ }^{31}$ (A) Heat of dilution measurement. A total of I.4 $\mathrm{nL}$ of I4.7\% methanol-water solution was mixed with $2.1 \mathrm{~nL}$ of water. (B) The measurement time is limited by the mixing time, typically via diffusion. 
because the evaporation of the sample due to heating will adversely affect the DSC measurements. Two different chip device configurations have been demonstrated for DSC operations: the sealed-chamber and flow-through channel configurations. In fact, the DSC and flow operations have been demonstrated with identical devices. ${ }^{35,36}$ As PDMS has fairly large permeability on water vapor, glass or polymers impermeable to water, such as SU-8 and parylene, are recommended for the construction of the chamber. The temperature and pressure ranges within which the DSC can operate are also important factors to consider. Generally, the polymer microfluidic channels become unstable at high temperatures and pressures. Using etched-glass microfluidic channels, high-temperature operations (up to $800^{\circ} \mathrm{C}$ ) have been conducted with chip calorimeters. ${ }^{37,39}$ Applying high pressures extends the temperature-scanning range by increasing the boiling temperature of water. There is no simple way of making a high-pressure valve for microfluidic systems. Sealing the fluidic channel using epoxy has been suggested for DSC operation up to $\sim 250^{\circ} \mathrm{C}$ with liquid samples, ${ }^{37}$ but actual measurements have not been reported. DSC operation will be discussed further in the Applications section.

\section{Applications}

Conventional calorimeters have long been refined and optimized for specific applications while the development of chip calorimeters is still in its infancy. Chip calorimeters have power sensitivities similar to or slightly better than those of conventional calorimeters $(\sim 10-100 \mathrm{nW})$, but the sample volumes of chip calorimeters are much smaller (up to $\left.1 / 10^{5}\right)$. As the volume-specific heat power sensitivity (power sensitivity divided by the sample volume) of chip calorimeters is degraded by decreasing the sample volume,${ }^{63}$ chip calorimeters require samples with higher concentrations or densities. The structural strength and the sample-handling methods also prohibit certain application types, such as the use of volatile samples and operation at a high pressure. These limit the applications of chip calorimeters, as opposed to many applications of conventional calorimeters. Chip calorimeters, however, are useful and promising in several application areas due to their fast response and small size. Moreover, they are expected to become even more powerful with the improvement of their sensitivity and fluid-handling capability through the use of new technologies. Currently, chip calorimeters are being developed for four major application areas: high-throughput calorimetry, DSCs, biochemical sensing, and cellular activity monitoring.

\section{High-throughput characterization of biochemical interactions}

The thermodynamic characterization of reactions is one of the most important application areas of calorimeters. Both modern industry and fundamental science require approaches that involve large libraries of compounds, ranging from small molecules to proteins and other macromolecules. ${ }^{64,65}$ High-throughput calorimeters can have a great impact on many areas, including biochemical reaction libraries and the drug discovery industry. ${ }^{8,66}$

In principle, all chip calorimeters can be operated in parallel to increase their measurement throughput. The fluid-handling methods, however, do not always support highthroughput measurements with array operations. As discussed in the previous section, fluid delivery for open-chamber chip calorimeters has a high level of noise from the evaporation and kinetic energy. One way of circumventing this problem is increasing the signal by increasing the sample volume. A large-volume $(\sim 10-600 \mu \mathrm{L})$ reaction chamber and $\sim 1-\mu \mathrm{L}$ substrate injection have been used for microwell-type calorimeter arrays. ${ }^{23,24}$ The better solution, which involved sacrificing small sample volumes, was using electrostatic droplet merging, as previously described. ${ }^{22}$ The enthalpy array using electrostatic merging enabled high-throughput heat-of-reaction measurements (96 measurements in parallel) with $500 \mathrm{~nL}$ samples. For closed-chamber chip calorimeters, substantial heat loss and inaccuracy of the sample injection volume have been issues. It is difficult for the typical closed-chamber chip calorimeter to make an accurate sample injection because its large-scale tubing and pumps result in a large dead volume. Fluid handling with on-chip valves and pumps has been reported to show relatively high accuracy in sample volume control ( $7 \%$ error for 0.7 -nL injection). ${ }^{31}$ The successful measurement of the heat of reaction with a few $\mathrm{nL}$ samples has been reported. The system could be multiplexed without much complication, but no array operation has been demonstrated to date.

\section{DSC with a minimal sample volume}

DSCs are widely used for studying protein folding, and it is well known that the development of several disorders, such as Alzheimer's and Parkinson's diseases, is a consequence of the misfolding of proteins. Small chip calorimeters can be particularly advantageous because their small heat capacity necessitates a small sample volume, which results in a fast scanning rate. The small sample volume requirement also results in a lower cost and enables the measurement of a scarce sample, such as proteins produced by the 
subpopulation of a heterogeneous cell culture. Several groups have demonstrated the use of scanning chip calorimeters with $10-\mu \mathrm{g}$ biological samples. ${ }^{17,21,28,36}$ Considering that the typical commercial DSC with a much higher device heat capacity requires similar amounts of samples, the sensitivity of the chip DSCs still needs to be improved. As discussed in the previous section, there are also technical difficulties in building a structure that can withstand high temperatures and pressures while maintaining high sensitivity.

\section{Label-free biochemical sensor}

Chip calorimeters can use various recognition elements as biosensors: enzymes, antibodies, DNA, cells, etc. The biochemical detection of a specific substrate is carried out by measuring the heat of reaction. The simplest method for this is making a co-flow of the substrate and the antibody or enzyme, which are mixed by diffusion in a reaction chamber. ${ }^{30,34,42,43}$ This method, however, will result in the large consumption of antibodies or enzymes. Immobilization of the recognition elements is thus preferable to reduce the material cost and long running time. The enzymes immobilized on microbeads can be loaded into a chamber or directly deposited in the chamber to detect substrates..$^{27,35,38,40,41}$ Similarly, antibodies or DNA can be used for calorimetric sensing. ${ }^{26}$ DNA "thermosequencing" ${ }^{67}$ using a chip calorimeter can be achieved in this manner. Packing microbeads in the reaction chamber is a convenient way of increasing the surface area for biomolecule binding. In addition, microbeads can be coated with antibodies outside the calorimeter chip, and can be introduced into the reaction chamber, which will help localize the heat generation in the chamber. Calorimetric biochemical sensors have the advantages of real-time, label-free sensing for long-term use without sensor element fouling.

\section{Bioprocess monitoring}

A physiometer is a device that measures the activities of living cells. The activities or metabolism of living cells can be quantified in several ways, such as by measuring the concentrations of the analytes (eg, glucose and adenosine5 -triphosphate). The changes in the metabolic rate as a form of released heat can be detected real-time with calorimetric measurements, which can give an important insight into the dynamics of the underlying biological processes. The calorimetric study of cellular metabolism is not yet a well-recognized field, mainly due to the lack of specialized equipment. A chip calorimeter has great potential as an analytical tool for measuring cellular metabolism. The microfluidic capability can provide a controllable environment for various cell types, and calorimetric measurement is label-free and nondestructive and is thus compatible with other analysis methods. Perfused media can be analyzed for specific analyte concentrations downstream and harvested cells and cell contents can be analyzed using other techniques, such as protein chips and gel electrophoresis.

Measurements of metabolic rate changes using chip calorimeters have been demonstrated for various cell types, including bacteria, ${ }^{29,32}$ mammalian cells (Figure 4), ${ }^{12,23}$ and fish embryos. ${ }^{33}$ Such studies have shown the possibility

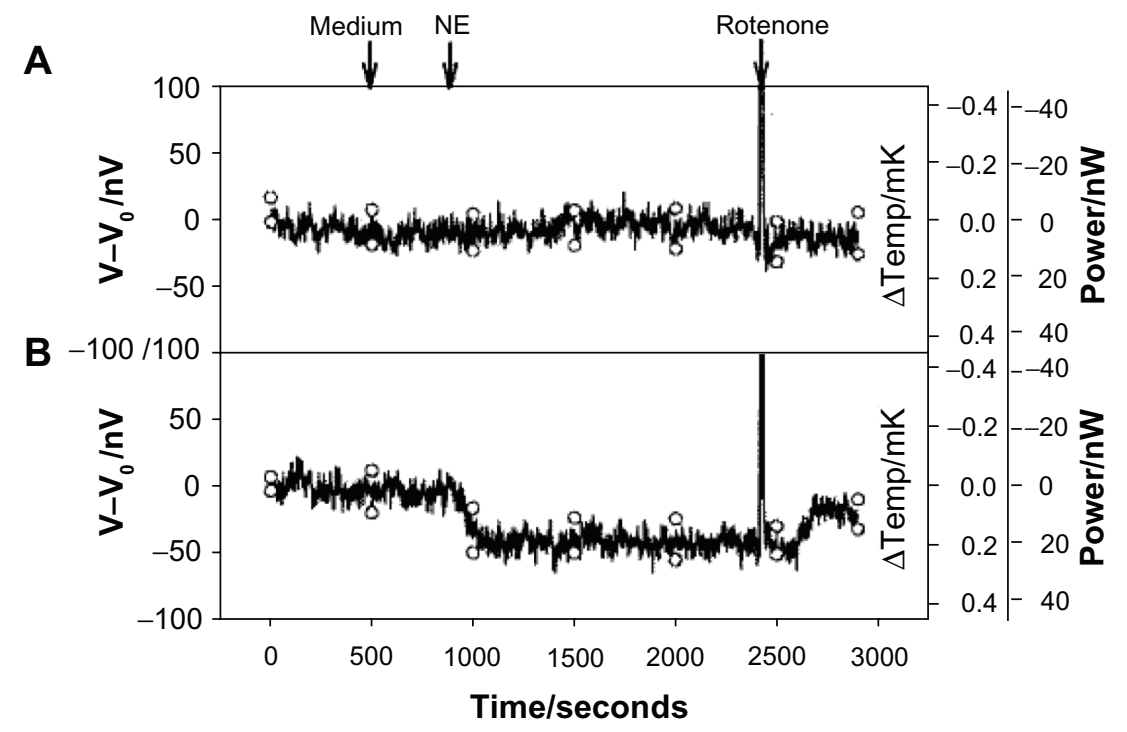

Figure 4 Thermogenic response of brown adipocytes to norepinephrine. (A) Control experiments without cells. (B) Data with 10 brown adipocytes. Copyright (C) 2002, ACS Publications. Reproduced with permission from Johannessen EA, Weaver JMR, Bourova L, Svoboda P, Cobbold PH, Cooper JM. Micromachined nanocalorimetric sensor for ultra-low-volume cell-based assays. Anal Chem. 2002;74(9):2190-2197. Copyright (2002) American Chemical Society. ${ }^{12}$ Chip calorimeters enable the quantitative measurement of the activities of cells. 
of using chip calorimeters as physiometers by monitoring metabolic rate changes based on the effects of various stimuli, such as antibiotics ${ }^{32,50}$ and hormones. ${ }^{12,23}$ The growth of suspended bacteria ${ }^{29}$ and a biofilm ${ }^{68}$ within a chip calorimeter has been observed, but long-term measurement with the on-chip cell culture of mammalian cells has not been demonstrated, mainly due to the decrease in sensitivity with the integration of such microfluidic capabilities.

The combination of a flow-through calorimeter with a bioreactor system is also an actively studied method. The chip calorimeter allows the online monitoring of the microbiological process with a short measurement loop that allows a fast response. There are several well-written review papers regarding this research area. ${ }^{69-71}$

\section{Outlook and conclusion}

Chip calorimeters have potential as unique and powerful biosensors, but their low sensitivity due to their large heat loss and awkwardness in fluid handling have been major hurdles impeding advancement in chip calorimeter technology. Diverse microfluidic components, such as mixing, pumping, and droplet microfluidics, ${ }^{72,73}$ may be adopted for the fluid handling of chip calorimeters, which will greatly improve their applicability. Applying electrostatic droplet-merging technology for the enthalpy array is a good example of this. ${ }^{22}$ Vacuum-insulated microfluidic systems are expected to enable further major improvements in sensitivity. ${ }^{31}$ In particular, vacuum insulation can allow chip calorimeters to achieve a very important sensitivity range: $\sim 10 \mathrm{pW}$ or the single-cell metabolic rate. The average basal metabolic rate of a single mammalian cell is $\sim 60 \mathrm{pW} .{ }^{74}$ A calorimeter with 1-10 pW sensitivity will be able to resolve the metabolic rate change of a single cell.

The traditional methods of studying cellular metabolism depend heavily on measuring the ensemble average of cells or extracts. The resulting averaged value is often misleading, however, because it does not always represent individual cellular behavior. Moreover, the concentration step results in a delay in measurement. The direct measurement of the metabolic rate of a single cell is expected to help answer many important biological questions. For example, cancerous cells are known to have higher metabolic rates than normal cells. Thus, positron emission tomography scans can find cancerous tissues or organs by detecting the regions with high glucose uptake. Calorimetric measurements also confirm that the metabolic powers of various cancerous tissues are higher than those of normal cells. ${ }^{75}$ The measurements, however, are from the ensemble average of a large number of cells, and little is known about the metabolism of individual cells, although heterogeneity is important for understanding cancer. Tumor biologists and oncologists typically seek to identify the genetic and molecular changes that distinguish normal cells from tumor cells, and to develop therapeutic agents that can exploit these differences. However, there are other movements among physical scientists and engineers to understand cancer, using totally different approaches. ${ }^{76}$ They want to understand cancer by investigating different parameters, such as energetics, entropy, and mechanical forces. Chip calorimeters are expected to help in quantifying the metabolic changes arising from various cues that influence cancer cell metabolism. Apart from this, chip calorimeters can also be used to identify and detect cancer cells (eg, biopsy samples or circulating tumor cells). Ultimately, they can be integrated with other sensors to build a micro total analysis system, not only for fundamental cell biology research ${ }^{77}$ but also for point-of-care cancer diagnosis. ${ }^{78}$

As the process of bringing a candidate drug from conception to the market takes $12-15$ years and entails an average cost of around US\$1.8 billion, failure at the latter stage of drug discovery due to toxicity is very costly. It is hard to predict the effect of a drug on an organism using a biochemical assay because the response is complex and involves interactions at multiple levels; therefore, cell-based screening assays are being increasingly used as more biologically relevant surrogates. Compared to the existing cell-based assays, calorimeters can provide easier, faster, and more reliable methods because their metabolic measurements are label-free, real-time, and free from user bias. It is expected that high-sensitivity microfluidic calorimeters will provide a new standard for cell-based assays, especially in terms of single-cell sensitivity.

Chip calorimeters are attracting growing interest due to the development of new capabilities, such as high-throughput array operations and vacuum-insulated microfluidics. In this review, diverse approaches for fabricating chip calorimeters, and their applications, are discussed. Efforts to construct and demonstrate the operation of chip calorimeters are converging on the search for diverse applications. The unique capability of calorimetric sensing is expected to open an unconventional biosensor field, and the improved sensitivity and throughput of chip calorimeters will allow many new applications.

\section{Disclosure}

The authors report no conflicts of interest in this work. 


\section{References}

1. Fabjan CW, Ludlam T. Calorimetry in high-energy physics. Annu Rev Nucl Sci. 1982;32:335-389.

2. Ladbury JE, Klebe G, Freire E. Adding calorimetric data to decision making in lead discovery: a hot tip. Nat Rev Drug Discov. 2010;9(1): 23-27.

3. Chaires JB. Calorimetry and thermodynamics in drug design. Annu Rev Biophys. 2008;37:135-151.

4. Jelesarov I, Bosshard HR. Isothermal titration calorimetry and differential scanning calorimetry as complementary tools to investigate the energetics of biomolecular recognition. J Mol Recognit. 1999;12(1):3-18.

5. Judy JW. Microelectromechanical systems (MEMS): fabrication, design and applications. Smart Mater Struct. 2001;10(6):1115-1134.

6. Manz A, Graber N, Widmer HM. Miniaturized total chemical analysis systems: A novel concept for chemical sensing. Sens Actuators B Chem. 1990;1(1-6):244-248

7. Voldman J, Gray ML, Schmidt MA. Microfabrication in biology and medicine. Annu Rev Biomed Eng. 1999;1:401-425.

8. Torres FE, Recht MI, Coyle JE, Bruce RH, Williams G. Higher throughput calorimetry: opportunities, approaches and challenges. Curr Opin Struct Biol. 2010;20(5):598-605.

9. Chancellor EB, Wikswo JP, Baudenbacher F, Radparvar M, Osterman D. Heat conduction calorimeter for massively parallel high throughput measurements with picoliter sample volumes. Appl Phys Lett. 2004;85(12):2408-2410.

10. Hemminger W, Höhne G. Calorimetry: Fundamentals and Practice. Weinheim, Germany: Verlag Chemie; 1984.

11. van Herwaarden S. Physical principles of thermal sensors. Mater Sci Eng C Biomim Mater Sens Syst. 1996;8(6):373-387.

12. Johannessen EA, Weaver JMR, Bourova L, Svoboda P, Cobbold PH, Cooper JM. Micromachined nanocalorimetric sensor for ultra-lowvolume cell-based assays. Anal Chem. 2002;74(9):2190-2197.

13. Johannessen EA, Weaver JMR, Cobbold PH, Cooper JM. A suspended membrane nanocalorimeter for ultralow volume bioanalysis. IEEE Trans Nanobioscience. 2002;1(1):29-36.

14. Johannessen EA, Weaver JMR, Cobbold PH, Cooper JM. Heat conduction nanocalorimeter for pl-scale single cell measurements. Appl Phys Lett. 2002;80(11):2029-2031.

15. Kim J-K, Kim T-H, Cho S-C, Shin S-M, Lee S-K. Modeling and fabrication of thin film thermopile sensor. J Vac Sci Technol B Microelectron Nanometer Struct. 2009;27(3):1466-1472.

16. Lubbers B, Baudenbacher F. Isothermal titration calorimetry in canoliter droplets with subsecond time constants. Anal Chem. 2011;83(20): 7955-7961.

17. Olson EA, Efremov MY, Kwan AT, et al. Scanning calorimeter for nanoliter-scale liquid samples. Appl Phys Lett. 2000;77(17):2671-2673.

18. Podzemna V, Slovakova M, Kourkova L, Svoboda L. Utilization of the IC-calorimeter for study of enzymatic reaction. JTherm Anal Calorim. 2010;101(2):715-719.

19. Recht MI, De Bruyker D, Bell AG, et al. Enthalpy array analysis of enzymatic and binding reactions. Anal Biochem. 2008;377(1):33-39.

20. Recht MI, Torres FE, De Bruyker D, Bell AG, Klumpp M, Bruce RH. Measurement of enzyme kinetics and inhibitor constants using enthalpy arrays. Anal Biochem. 2009;388(2):204-212.

21. Svanidze AV, Huth H, Lushnikov SG, Kojima S, Schick C. Phase transition in tetragonal hen egg-white lysozyme crystals. Appl Phys Lett. 2009;95(26):263702.

22. Torres FE, Kuhnt P, De Bruyker D, et al. Enthalpy arrays. Proc Natl Acad Sci U S A. 2004;101(26):9517-9522.

23. Verhaegen K, Baert K, Simaels J, Van Driessche W. A high-throughput silicon microphysiometer. Sens Actuators A Phys. 2000;82(1-3): 186-190.

24. Vermeir S, Nicolai BM, Verboven P, et al. Microplate differential calorimetric biosensor for ascorbic acid analysis in food and pharmaceuticals. Anal Chem. 2007;79(16):6119-6127.
25. Xu J, Reiserer R, Tellinghuisen J, Wikswo JP, Baudenbacher FJ. A microfabricated nanocalorimeter: Design, characterization, and chemical calibration. Anal Chem. 2008;80(8):2728-2733.

26. Ahmad LM, Towe B, Wolf A, Mertens F, Lerchner J. Binding event measurement using a chip calorimeter coupled to magnetic beads. Sens Actuators B Chem. 2010;145(1):239-245.

27. Bataillard P, Steffgen E, Haemmerli S, Manz A, Widmer HM. An integrated silicon thermopile as biosensor for the thermal monitoring of glucose, urea and penicillin. Biosens Bioelectron. 1993;8(2):89-98.

28. Garden JL, Chateau E, Chaussy J. Highly sensitive ac nanocalorimeter for microliter-scale liquids or biological samples. Appl Phys Lett. 2004;84(18):3597-3599.

29. Higuera-Guisset J, Rodriguez-Viejo J, Chacon M, Munoz FJ, Vigues N, Mas J. Calorimetry of microbial growth using a thermopile based microreactor. Thermochim Acta. 2005;427(1-2):187-191.

30. Kwak BS, Kim BS, Cho HH, Park JS, Jung HI. Dual thermopile integrated microfluidic calorimeter for biochemical thermodynamics. Microfluid Nanofluidics. 2008;5(2):255-262.

31. Lee W, Fon W, Axelrod BW, Roukes ML. High-sensitivity microfluidic calorimeters for biological and chemical applications. Proc Natl Acad Sci US A. 2009;106(36):15225-15230.

32. Lerchner J, Mueller-Hagen D, Roehr H, et al. Chip-calorimetric evaluation of the efficacy of antibiotics and bacteriophages against bacteria on a minute-timescale. J Therm Anal Calorim. 2011;104(1):31-36.

33. Lerchner J, Wolf A, Schneider HJ, et al. Nano-calorimetry of smallsized biological samples. Thermochim Acta. 2008;477(1-2):48-53.

34. Lerchner J, Wolf A, Wolf G, et al. A new micro-fluid chip calorimeter for biochemical applications. Thermochim Acta. 2006;445(2):144-150.

35. Li W, Sipe DM, Yong X, Qiao L. A MEMS thermal biosensor for metabolic monitoring applications. J Microelectromech Syst. 2008;17(2): 318-327.

36. Li Wang, Bin Wang, Qiao Lin. Demonstration of MEMS-based differential scanning calorimetry for determining thermodynamic properties of biomolecules. Sens Actuators B Chem. 2008;134(2):953-958.

37. Liu YS, Ugaz VM, Rogers WJ, Mannan MS, Saraf SR. Development of an advanced nanocalorimetry system for material characterization. Journal of Loss Prevention in the Process Industries. 2005;18(3): 139-144.

38. van Herwaarden AW, Sarro PM, Gardner JW, Bataillard P. Liquid and gas micro-calorimeters for (bio)chemical measurements. Sens Actuators A Phys. 1994;43(1-3):24-30.

39. Vereshchagina E, Wolters RAM, Gardeniers JGE. Measurement of reaction heats using a polysilicon-based microcalorimetric sensor. Sens Actuators A Phys. 2011;169(2):308-316.

40. Xie B, Mecklenburg M, Danielsson B, Ohman O, Norlin P, Winquist F. Development of an integrated thermal biosensor for the simultaneous determination of multiple analytes. Analyst. 1995;120(1):155-160.

41. Xie B, Mecklenburg M, Danielsson B, Ohman O, Winquist F. Microbiosensor based on an integrated thermopile. Anal Chim Acta. 1994; 299(2):165-170.

42. Yoon S-I, Park S-C, Kim Y-J. A micromachined microcalorimeter with split-flow microchannel for biochemical sensing applications. Sens Actuators B Chem. 2008;134(1):158-165.

43. Zhang YY, Tadigadapa S. Calorimetric biosensors with integrated microfluidic channels. Biosens Bioelectron. 2004;19(12):1733-1743.

44. Noh HS, Huang Y, Hesketh PJ. Parylene micromolding, a rapid and low-cost fabrication method for parylene microchannel. Sens Actuators B Chem. 2004;102(1):78-85.

45. Xie J, Miao YN, Shih J, et al. An electrochemical pumping system for on-chip gradient generation. Anal Chem. 2004;76(13):3756-3763.

46. Burg TP, Godin M, Knudsen SM, et al. Weighing of biomolecules, single cells and single nanoparticles in fluid. Nature. 2007;446(7139): 1066-1069.

47. Wang L, Sipe DM, Xu Y, Lin Q. A MEMS thermal biosensor for metabolic monitoring applications. J Microelectromech Syst. Apr 2008; 17(2):318-327. 
48. Ahmad LM, Towe B, Wolf A, Mertens F, Lerchner J. Binding event measurement using a chip calorimeter coupled to magnetic beads. Sens Actuators B Chem. 2010;145(1):239-245.

49. Baier V, Fodisch R, Ihring A, et al. Highly sensitive thermopile heat power sensor for micro-fluid calorimetry of biochemical processes. Sens Actuators A Phys. 2005;123-24:354-359.

50. Buchholz F, Wolf A, Lerchner J, Mertens F, Harms H, Maskow T. Chip calorimetry for fast and reliable evaluation of bactericidal and bacteriostatic treatments of biofilms. Antimicrob Agents Chemother. 2010;54(1):312-319.

51. Lerchner J, Wolf A, Wolf G. Recent developments in integrated circuit calorimetry. J Therm Anal Calorim. 1999;57(1):241-251.

52. Lerchner J, Wolf A, Wolf G, Fernandez I. Chip calorimeters for the investigation of liquid phase reactions: design rules. Thermochim Acta. 2006;446(1-2):168-175.

53. Peitzsch M, Kiesel B, Harms H, Maskow T. Real time analysis of Escherichia coli biofilms using calorimetry. Chem Eng Process. 2008;47(6):1000-1006.

54. Zhang YY, Tadigadapa S. Thermal characterization of liquids and polymer thin films using a microcalorimeter. Appl Phys Lett. 2005;86(3).

55. Black CM, Clark RP, Darton K, Goff MR, Norman TD, Spikes HA. A pyroelectric thermal imaging system for use in medical diagnosis. J Biomed Eng. 1990;12(4):281-286.

56. Paulik MA, Buckholz RG, Lancaster ME, et al. Development of infrared imaging to measure thermogenesis in cell culture: thermogenic effects of uncoupling protein-2, troglitazone, and $\beta$-adrenoceptor agonists. Pharm Res. 1998;15(6):944-949.

57. Swinney K, Bornhop DJ. Quantification and evaluation of Joule heating in on-chip capillary electrophoresis. Electrophoresis. 2002;23(4): 613-620.

58. Pramanik M, Wang LV. Thermoacoustic and photoacoustic sensing of temperature. J Biomed Opt. 2009;14(5):054024-054027.

59. Irina VL, Kirill VL, Rinat OE. Real-time optoacoustic monitoring of temperature in tissues. J Phys D Appl Phys. 2005;38(15):2633.

60. David R, Hunter IW. A liquid expansion microcalorimeter. J Therm Anal Calorim. 2007;90(2):597-599.

61. David R, Hunter IW. A liquid-in-glass thermometer read by an interferometer. Sens Actuators A Phys. 2005;121(1):31-34.

62. Gosse C, Bergaud C, Löw P. Molecular probes for thermometry in microfluidic devices thermal nanosystems and nanomaterials. In: Volz S, editor. Thermal Nanosystems and Nanomaterials. Berlin, Germany: Springer; 2009:301-341. Topics in Applied Physics, Vol. 118.

63. Lerchner J, Maskow T, Wolf G. Chip calorimetry and its use for biochemical and cell biological investigations. Chem Eng Process. 2008;47(6):991-999.

64. de Wildt RMT, Mundy CR, Gorick BD, Tomlinson IM. Antibody arrays for high-throughput screening of antibody-antigen interactions. Nat Biotechnol. 2000;18(9):989-994.

65. Bleicher KH, Bohm HJ, Muller K, Alanine AI. Hit and lead generation: beyond high-throughput screening. Nat Rev Drug Discov. 2003;2(5):369-378.

66. Salemme FR. High-throughput biochemistry heats up. Nat Biotechnol. 2004;22(9):1100-1101.

67. Esfandyarpour H, Zheng B, Pease RFW, Davis RW. Structural optimization for heat detection of DNA thermosequencing platform using finite element analysis. Biomicrofluidics. 2008;2(2):024102-024111.
68. Lerchner J, Wolf A, Buchholz F, et al. Miniaturized calorimetry - A new method for real-time biofilm activity analysis. $J$ Microbiol Methods. 2008;74(2-3):74-81.

69. Maskow T, Schubert T, Wolf A, et al. Potentials and limitations of miniaturized calorimeters for bioprocess monitoring. Appl Microbiol Biotechnol. 2011;92(1):55-66.

70. Maskow T, Harms H. Real time insights into bioprocesses using calorimetry: state of the art and potential. Engineering in Life Sciences. 2006;6(3):266-277.

71. Kemp R. 'Gie me ae spark o' nature's fire'. An insight into cell physiology from calorimetry. J Therm Anal Calorim. 2000;60(3):831-843.

72. Stone HA, Stroock AD, Ajdari A. Engineering flows in small devices: microfluidics toward a lab-on-a-chip. Annu Rev Fluid Mech. 2004;36:381-411.

73. Ng JMK, Gitlin I, Stroock AD, Whitesides GM. Components for integrated poly(dimethylsiloxane) microfluidic systems. Electrophoresis. 2002;23(20):3461-3473.

74. West GB, Woodruff WH, Brown JH. Allometric scaling of metabolic rate from molecules and mitochondria to cells and mammals. Proc Natl Acad Sci U S A. 2002;99:2473-2478.

75. Karnebogen M, Singer D, Kallerhoff M, Ringert RH. Microcalorimetric investigations on isolated tumorous and nontumorous tissue samples. Thermochim Acta. 1993;229:147-155.

76. Cancer.gov [homepage on the Internet]. Office of Physical SciencesOncology. Bethesda, MD: National Cancer Institute. Available from: http://physics.cancer.gov.

77. El-Ali J, Sorger PK, Jensen KF. Cells on chips. Nature. 2006;442(7101): 403-411.

78. Rasooly A, Jacobson J. Development of biosensors for cancer clinical testing. Biosens Bioelectron. 2006;21(10):1851-1858.

79. Material Property Database [homepage on the Internet]. LPCVD Silicon Nitride. Available from: http://www.mit.edu/ 6.777/matprops/ lpcvd_sin.htm. Accessed on March 19, 2012.

80. Glassbrenner CJ, Slack GA. Thermal conductivity of silicon and germanium from $3^{\circ} \mathrm{K}$ to the melting point. Phys Rev. 1964;134(4A): A1058-A1069.

81. Okhotin AS, Pushkarskii AS, Gorbachev VV. Thermophysical Properties of Semiconductors. Moscow, Russia: Atomizdat; 1972. Russian.

82. L.H Martin KCL. Thermal conductivity of water. Proc Phys Soc. 1933;45:523.

83. Mark JE, editor. Polymer Data Handbook. New York, NY: Oxford University Press; 1999.

84. DuPont.com [homepage on the Internet]. Dupont Kapton Polyimide Film General Specifications. Available from: http:/www2.dupont.com/ Kapton/en_US/assets/downloads/pdf/summaryofprop.pdf. Accessed February 10, 2012.

85. Paryleneengineering.com [homepage on the Internet]. Typical specification of Parylene C. Redmond, WA: Parylene Engineering. Available from: http://www.paryleneengineering.com/specifications_parylene_c_ astm.html. Accessed March 19, 2012.

86. Engineeringtoolbox.com [homepage on the Internet]. Air properties. The Engineering ToolBox. Available from: http://www.engineeringtoolbox. com/air-properties-d_156.html. Accessed March 19, 2012.

87. Shackelford JF, Alexander W. CRC Materials Science and Engineering Handbook. 3rd ed. New York, NY: CRC Press; 2001.

88. Mills AF. Basic Heat and Mass Transfer. 2nd ed. Upper Saddle River, NJ: Prentice Hall; 1999.

Nanobiosensors in Disease Diagnosis

\section{Publish your work in this journal}

Nanobiosensors in Disease Diagnosis is an international, peer-reviewed, open access journal publishing original research, reports, reviews and commentaries including but not confined to: Diagnosis of diseases including cancer, cardiovascular, infectious diseases; Molecular modelling in diagnosis; Enzyme and membrane technologies; and quantum

dot fluorescence technologies for monitoring toxins and pathogens. The manuscript management system is completely online and includes a very quick and fair peer-review system, which is all easy to use. Visit http://www.dovepress.com/testimonials.php to read real quotes from published authors. 